TITLE:

\title{
Dynamical Time-Reversal and Inversion Symmetry Breaking, Dimensional Crossover, and Chiral Anomaly in a-(BEDT-TTF)।
}

\author{
$\operatorname{AUTHOR}(S)$ :
}

Morinari, Takao

\section{CITATION:}

Morinari, Takao. Dynamical Time-Reversal and Inversion Symmetry Breaking, Dimensional Crossover, and Chiral Anomaly in a-(BEDT-TTF)I. Journal of the Physical Society of Japan 2020, 89(7): 073705.

ISSUE DATE:

2020-07-15

URL:

http://hdl.handle.net/2433/254357

\section{RIGHT:}

(C2020 The Physical Society of Japan; The full-text file will be made open to the public on 11 June 2021 in accordance with publisher's 'Terms and Conditions for Self-Archiving'.; こ の論文は出版社版でありません。引用の際には出版社版をご確認ご利用ください。;

This is not the published version. Please cite only the published version. 


\title{
Dynamical Time-Reversal and Inversion Symmetry Breaking, Dimensional Crossover, and Chiral Anomaly in $\alpha$-(BEDT-TTF $)_{2} \mathbf{I}_{3}$
}

\author{
Takao Morinari* \\ Graduate School of Human and Environmental Studies, Kyoto University, Kyoto 606-8501, Japan
}

(Dated: June 11, 2020)

\begin{abstract}
In most Dirac semimetals, time-reversal and inversion symmetries are believed to play a crucial role in their stability. We demonstrate that these symmetries are broken in Dirac fermions in the organic conductor $\alpha$-(BEDT-TTF $)_{2} \mathrm{I}_{3}$ due to the bond correlation created by the strong inter-site electronic correlation. The system is a three-dimensional type-II Dirac semimetal in the coherent inter-layer tunneling regime. A chiral anomaly is predicted to be observed in the magnetoresistance when the magnetic field is tuned to the inter-layer tunneling direction. Our result suggests that $\alpha$-(BEDT-TTF $)_{2} \mathrm{I}_{3}$ is a useful platform to explore interplay between the chiral anomaly and the strong correlation and/or dimensionality.
\end{abstract}

Recently, the topological Dirac and Weyl semimetals have attracted intense theoretical and experimental interest because of their intriguing topological and electronic properties. ${ }^{1,2}$ In nodal semimetals, the conduction and valence bands touch only at certain points in the Brillouin zone (BZ), and the low-energy excitations are described by a relativistic Dirac or Weyl equation, where the velocity of light is replaced by the Fermi velocity. ${ }^{3}$ Two-dimensional (2D) Dirac semimetals are realized in graphene,${ }^{4}$ as is clearly demonstrated by the Dirac nature of the electronic transport, and the surface state of the three-dimensional (3D) topological insulators, ${ }^{5,6}$ where spin-orbit coupling plays a significant role. Based on theoretical and experimental efforts, 3D Dirac semimetals are now realized experimentally, for instance, in $\mathrm{Na}_{3} \mathrm{Bi}^{7,8}$ and $\mathrm{Cd}_{3} \mathrm{As}_{2} .{ }^{9,10}$ Remarkably, the topological semimetals have deep connections with particle physics because they provide solid state analogues of relativistic chiral fermions ${ }^{2,11}$ and lattice realizations ${ }^{12}$ of the chiral anomaly of the quantum field theory. ${ }^{13,14}$ Experimental evidence has been accumulated about the existence of Fermi arc surface states ${ }^{15-18}$ and the novel responses of the chiral anomaly to applied electronic and magnetic fields. ${ }^{19-23}$

Now it is well accepted that symmetries and spin-orbit coupling are the keys to realizing 3D Dirac semimetals in general. Spin-orbit coupling can create a linear energy dispersion and a 3D Dirac semimetal appears when the symmetry conditions are met. Since the net Chern numbers at each contact point are zero, we need additional crystal symmetries. ${ }^{7,9,24-26}$ In such 3D Dirac semimetal systems, Dirac points are on the symmetry lines in the BZ. Our current understanding of the condition of the Dirac semimetal is mostly based on symmetry consideration and spin-orbit coupling, though the latter is not necessary for some exceptional cases. ${ }^{27}$

In this Letter, we demonstrate that the quasi-2D organic conductor, $\alpha$-(BEDT-TTF $)_{2} \mathrm{I}_{3}$, is an unprecedented type of Dirac semimetal that does not fit into our current understanding of Dirac semimetal conditions. We show that contrary to other Dirac semimetals, both the time-reversal symmetry (TRS) and inversion sym- metry are broken and spin-orbit coupling plays no role. In the symmetry broken state, the energies of the Dirac points are shifted asymmetrically from the Fermi energy and their positions are not symmetrically located with respect to the origin of the BZ. We also show that the system exhibits a dimensional crossover from a type-I 2D Dirac semimetal to a type-II 3D Dirac semimetal ${ }^{28}$ upon entering the coherent inter-layer tunneling regime.

The organic conductor $\alpha$-(BEDT-TTF $)_{2} \mathrm{I}_{3}$ with the space group $P \overline{1}$ is a $2 \mathrm{D}$ Dirac semimetal with a layered structure comprising of Dirac fermion layers and insulating layers. ${ }^{29}$ The Dirac points are at non-high-symmetry points in the BZ similar to certain inorganic materials with the $P \overline{1}$ space group. ${ }^{27,30}$ In $\alpha$-(BEDT-TTF $)_{2} \mathrm{I}_{3}$, the unit cell is composed of four BEDT-TTF molecules, where BEDT-TTF is bis(ethylenedithio)tetrathiafulvalene, A, A', B, and C, in the conduction layer, ${ }^{31,32}$ as shown in Fig. 1(a). The system is metallic above $135 \mathrm{~K}$ but undergoes a metalinsulator transition ${ }^{33-35}$ at $135 \mathrm{~K}$ where a charge order stripe pattern forms as confirmed by ${ }^{13} \mathrm{C}-\mathrm{NMR}$ (nuclear magnetic resonance) measurement. ${ }^{36}$ Here, the shortrange inter-site Coulomb repulsion plays a key role. ${ }^{37,38}$ The 2D Dirac semimetal appears when the charge order is suppressed under high-pressure as revealed by the tight-binding model calculation ${ }^{39}$ and confirmed by the first-principles calculations. ${ }^{40,41}$ It should be stressed that the band filling is fixed to $3 / 4^{32}$ and the Fermi energy is exactly at the Dirac point within these calculations. The inter-layer magnetoresistance, which is negative and is in inversely proportional to the applied magnetic field, clearly demonstrates the presence of the zeroenergy Landau level. ${ }^{42-45}$ Furthermore, the phase of the Dirac fermions is confirmed by the Shubnikov-de Haas oscillation of the hole-doped sample, where the sample is placed on polyethylene naphthalate substrate. ${ }^{46}$

Thus far, the presence of the massless Dirac fermion spectrum has been established in $\alpha$-(BEDT-TTF $)_{2} \mathrm{I}_{3}$ but the role of the strong electronic correlation is unclear: The charge order stripe pattern at ambient pressure is replaced by charge disproportionation, ${ }^{47,48}$ where $n_{\mathrm{A}}=$ $n_{\mathrm{A}^{\prime}}$ and $n_{\mathrm{B}} \neq n_{\mathrm{C}}$ with charge density at molecule $\alpha$ 

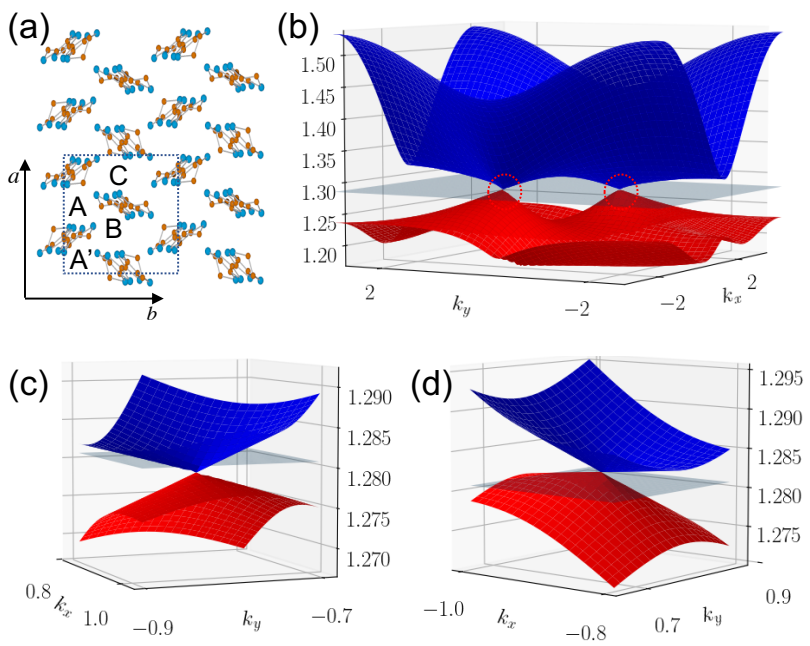

FIG. 1. (Color online) (a)Configuration of BEDT-TTF molecules in a conducting plane of $\alpha$-(BEDT-TTF $)_{2} \mathrm{I}_{3}$. The rectangle shows a unit cell that contains four molecules, A, $\mathrm{A}^{\prime}, \mathrm{B}$, and $\mathrm{C}$. The molecules stacked in the $a$ axis, which is taken as the $y$ axis and the $b$ axis is taken as the $x$ axis. (b) Conduction and valence bands are plotted as functions of $k_{x}$ and $k_{y}$ at $P=0.8$. There are two Dirac points at different energies, which are encircled by dotted circles, and they are located at the generic wave vectors. The horizontal plane denotes the Fermi energy. Magnified views of the Dirac nodes at $\boldsymbol{k}_{D}^{(1)}$ and $\boldsymbol{k}_{D}^{(2)}$ are shown in (c) and (d), respectively. The Dirac point at $\boldsymbol{k}_{D}^{(1)}$ is below the Fermi energy, while the Dirac point at $\boldsymbol{k}_{D}^{(2)}$ is above the Fermi energy.

denoted by $n_{\alpha}$. The on-site Coulomb repulsion plays a central role in the determination of the charge densities but the inter-site Coulomb interaction plays a minor role. The purpose of this study is to demonstrate that the inter-site Coulomb interaction plays a crucial role in stabilizing a non-trivial Dirac semimetal state.

The Hamiltonian of electrons within a conduction layer is given by $\mathcal{H}=\mathcal{H}_{0}+\mathcal{H}_{\text {int }}$. The first term describes the hopping between the molecules and in the momentum space,

$$
\mathcal{H}_{0}=\sum_{\boldsymbol{k}, \sigma=\uparrow, \downarrow} c_{\boldsymbol{k} \sigma}^{\dagger} \mathcal{H}_{\boldsymbol{k}}^{0} c_{\boldsymbol{k} \sigma}
$$

where $c_{\boldsymbol{k} \sigma}^{\dagger}=\left(c_{\boldsymbol{k} 1 \sigma}^{\dagger}, c_{\boldsymbol{k} 2 \sigma}^{\dagger}, c_{\boldsymbol{k} 3 \sigma}^{\dagger}, c_{\boldsymbol{k} 4 \sigma}^{\dagger}\right)$ is the fourcomponent creation operator for an electron with twodimensional momentum $k$ and spin component $\sigma$. The indices 1, 2, 3, and 4 represent molecules $\mathrm{A}, \mathrm{A}^{\prime}, \mathrm{B}$, and $\mathrm{C}$, respectively. The matrix elements of $H_{k}^{0}$ are $\left(\mathcal{H}_{\boldsymbol{k}}^{0}\right)_{12}=t_{a 3} e^{-i \boldsymbol{k} \cdot \boldsymbol{d}_{1}}+t_{a 2} e^{i \boldsymbol{k} \cdot \boldsymbol{d}_{1}},\left(\mathcal{H}_{\boldsymbol{k}}^{0}\right)_{13}=t_{b 3} e^{-i \boldsymbol{k} \cdot \boldsymbol{d}_{3}}+$ $t_{b 2} e^{i \boldsymbol{k} \cdot \boldsymbol{d}_{2}},\left(\mathcal{H}_{\boldsymbol{k}}^{0}\right)_{14}=t_{b 4} e^{-i \boldsymbol{k} \cdot \boldsymbol{d}_{2}}+t_{b 1} e^{i \boldsymbol{k} \cdot \boldsymbol{d}_{3}},\left(\mathcal{H}_{\boldsymbol{k}}^{0}\right)_{23}=$ $t_{b 2} e^{-i \boldsymbol{k} \cdot \boldsymbol{d}_{2}}+t_{b 3} e^{i \boldsymbol{k} \cdot \boldsymbol{d}_{3}},\left(\mathcal{H}_{\boldsymbol{k}}^{0}\right)_{24}=t_{b 1} e^{-i \boldsymbol{k} \cdot \boldsymbol{d}_{3}}+t_{b 4} e^{i \boldsymbol{k} \cdot \boldsymbol{d}_{2}}$, and $\left(\mathcal{H}_{\boldsymbol{k}}^{0}\right)_{34}=t_{a 1} e^{i \boldsymbol{k} \cdot \boldsymbol{d}_{1}}+t_{a 1} e^{-i \boldsymbol{k} \cdot \boldsymbol{d}_{1}}$, where the displacement vectors, $\boldsymbol{d}_{1}, \boldsymbol{d}_{2}$, and $\boldsymbol{d}_{3}$, respectively, are defined by $\boldsymbol{d}_{1}=(0, a / 2), \boldsymbol{d}_{2}=(b / 2,-a / 4)$, and $\boldsymbol{d}_{3}=(b / 2, a / 4) \cdot{ }^{29}$ Hereafter, we measure the energies in units of $\mathrm{eV}$ and we set $a=1$ and $b=1$ for the lattice constants. The transfer energies are pressure dependent and given by ${ }^{48}$ $t_{\alpha}=C_{\alpha}\left(1+b_{\alpha} P\right)$. Here, the pressure $P$ is in units of $\mathrm{GPa}$. The numerical coefficients $C_{\alpha}$ are $C_{a 1}=-0.028$, $C_{a 2}=0.048$, and $C_{a 3}=-0.020$ for the stacking direction and $C_{b 1}=0.123, C_{b 2}=0.140, C_{b 3}=-0.062$, and $C_{b 4}=-0.025$ for the other directions. The numerical constants $b_{\alpha}$ are $b_{a 1}=0.89, b_{a 2}=1.67, b_{a 3}=-0.25$, $b_{b 1}=0, b_{b 2}=0.11, b_{b 3}=0.32$, and $b_{b 4}=0$.

The interaction term $\mathcal{H}_{\text {int }}$ describes a strong electronic correlation that is given by

$$
\mathcal{H}_{\text {int }}=U \sum_{j, \alpha} n_{j \alpha \uparrow} n_{j \alpha \downarrow}+\sum_{i, j, \alpha, \beta} V_{i \alpha, j \beta} n_{i \alpha} n_{j \beta} .
$$

Here, the charge density of the electrons with spin $\sigma$ at molecule $\alpha$ in site $j$ is denoted by $n_{j \alpha \sigma}$, and we define $n_{j \alpha}=n_{j \alpha \uparrow}+n_{j \alpha \downarrow}$. The first term in the right-hand side describes the on-site Coulomb interaction while the second term describes the Coulomb interaction between nearest neighbor molecules. We set $V_{i 1, j 2}=V_{i 3, j 4}=V_{c}$ along the stacking direction of the BEDT-TTF molecules (the $a$-axis) and $V_{i \alpha, j \beta}=V_{p}$ otherwise. These interactions lead to an insulating state, which is a charge ordered state, ${ }^{37,38}$ under ambient pressure and lead to dynamical TRS and inversion symmetry breaking in the Dirac semimetal phase as we illustrate below.

It has not yet been considered explicitly, except for in limited cases, ${ }^{49,50}$ but the significant electronic correlation in the Dirac semimetal state, in the presence of the inter-molecule interaction $V_{\alpha \beta}$, is the bond correlation described by

$$
\chi_{\alpha \sigma, \beta \sigma^{\prime}, \pm}=\frac{1}{N} \sum_{\boldsymbol{k}} e^{-i \boldsymbol{k} \cdot \boldsymbol{d}_{\alpha \beta}^{( \pm)}}\left\langle c_{\boldsymbol{k} \alpha \sigma}^{\dagger} c_{\boldsymbol{k} \beta \sigma^{\prime}}\right\rangle,
$$

where $N$ is the number of BZ points and $\boldsymbol{d}_{13}^{(+)}=\boldsymbol{d}_{2}$, $\boldsymbol{d}_{13}^{(-)}=\boldsymbol{d}_{3}$, etc. Non-zero values of $\chi_{\alpha \sigma, \beta \sigma^{\prime}, \pm}$ can break the TRS and inversion symmetry. We also include the site order defined as

$$
n_{\alpha \sigma}=\frac{1}{N} \sum_{\boldsymbol{k}}\left\langle c_{\boldsymbol{k} \alpha \sigma}^{\dagger} c_{\boldsymbol{k} \alpha \sigma}\right\rangle
$$

which exhibits charge disproportionation in the Dirac semimetal. ${ }^{47,48}$ The resulting mean field Hamiltonian is denoted by

$$
\mathcal{H}_{\mathrm{mf}}=\sum_{\boldsymbol{k}, \sigma, \sigma^{\prime}} c_{\boldsymbol{k} \sigma}^{\dagger}[\mathcal{H}(\boldsymbol{k})]_{\sigma \sigma^{\prime}} c_{\boldsymbol{k} \sigma^{\prime}}
$$

The matrix $\mathcal{H}(\boldsymbol{k})$, with two-dimensional momentum $\boldsymbol{k}$, is $8 \times 8$ matrix. ${ }^{51}$ We solve the self-consistent equations for $\chi_{\alpha \sigma, \beta \sigma^{\prime}, \pm}$ and $n_{\alpha \sigma}$.

Now we describe symmetries of the system. To describe the symmetry operations, we define

$$
\mathcal{X}_{\mu \nu \lambda}=\sigma_{\mu} \otimes \sigma_{\nu} \otimes s_{\lambda}
$$


where $\mu, \nu, \lambda=0,1,2,3$. Here, $\sigma_{\mu}, \sigma_{\nu}$, and $s_{\lambda}$ denote the Pauli matrices and $\sigma_{0}$ and $s_{0}$ are the $2 \times 2$ unit matrix. In the definition of $\mathcal{X}_{\mu \nu \lambda}$, the first two Pauli matrices act on the four molecules and the last Pauli matrix acts on the spin index. Denoting the basis kets for the first two Pauli matrices by $\left|\sigma_{1}, \sigma_{2}\right\rangle$, the $\sigma_{1}=\uparrow$ state represents either the molecule $\mathrm{A}$ or $\mathrm{A}^{\prime}$ and the $\sigma_{1}=\downarrow$ state represents either the molecule B or C. For the former case, $\sigma_{2}=\uparrow$ $(\downarrow)$ represents the molecule $\mathrm{A}\left(\mathrm{A}^{\prime}\right)$. For the latter case, $\sigma_{2}=\uparrow(\downarrow)$ represents the molecule B (C). The TRS is

$$
\mathcal{X}_{002} \mathcal{H}^{*}(-\boldsymbol{k}) \mathcal{X}_{002}=\mathcal{H}(\boldsymbol{k}) .
$$

The system has an inversion center ${ }^{32,52,53}$ between $\mathrm{A}$ and $\mathrm{A}^{\prime}$. The symmetry operation associated with this inversion is

$$
\mathcal{I H}(-\boldsymbol{k}) \mathcal{I}=\mathcal{H}(\boldsymbol{k}),
$$

where $\mathcal{I}=i\left(\mathcal{X}_{103}+\mathcal{X}_{133}+\mathcal{X}_{003}-\mathcal{X}_{033}\right) / 2$. In the absence of the interactions, we see that the system is invariant under these symmetry operations. The situation does not change if we include the on-site Coulomb repulsion. If we restrict the self-consistent calculation to the Hartree level, these symmetries are unbroken but the exchange correlation associated with the inter-site Coulomb repulsion leads to breaking of both symmetries. We note that the combination of the inversion symmetry and TRS is broken as well. This is simply understood as the absence of the particle-hole symmetry, or charge conjugation that is clear from the asymmetric shifts of the Dirac point energies from the Fermi energy. This is in stark contrast to the other Dirac semimetal state with broken inversion symmetry and TRS but reserves their combination. ${ }^{54} \mathrm{We}$ also note that there is still spin degeneracy despite these symmetry breakings.

We present the energy dispersion of the conduction and valence bands in Fig. 1(b), (c), and (d). The conduction and valence bands are both spin degenerate. Contrary to the naive expectation, there is no mass gap in the Dirac fermion spectrum. We find that the two Dirac points are at $\boldsymbol{k}_{D}^{(1)}=(0.9250,-0.7978)$ where energy $\varepsilon_{D}^{(1)}=1.2788$ and at $\boldsymbol{k}_{D}^{(2)}=(-0.8988,0.7631)$ where en$\operatorname{ergy} \varepsilon_{D}^{(2)}=1.2822$. Here, the Fermi energy is $\varepsilon_{F}=1.2807$ and we note that $\boldsymbol{k}_{D}^{(1)} \neq-\boldsymbol{k}_{D}^{(2)}$ and $\varepsilon_{D}^{(1)}<\varepsilon_{F}<\varepsilon_{D}^{(2)}$. By changing the pressure, the Dirac points move in the BZ and the electronic correlation changes as well. Hereafter, we take $U=0.4, V_{c}=0.17, V_{p}=0.05$, and $P=0.8$. This set of interaction parameters reproduces the experimentally observed stripe pattern in the insulating state. ${ }^{48}$

Although the renormalized hopping parameters break the inversion symmetry, the charges do not. In fact, we find that $n_{\mathrm{A}}=n_{\mathrm{A}^{\prime}}=1.4554$ where $n_{\mathrm{B}}=1.2204$ and ${ }^{n} \mathrm{C}=1.8696$. This symmetry is protected by the strong correlation associated with $U$, whereas $V_{c}$ favors breaking this symmetry. The system undergoes a quantum phase transition as we increase the value of $V_{c}$, merging the two Dirac points. ${ }^{55}$
Now we investigate the dimensional crossover from the $2 \mathrm{D}$ electronic state to the $3 \mathrm{D}$ electronic state. Since $\alpha$-(BEDT-TTF $)_{2} \mathrm{I}_{3}$ has a layered structure and highmobility ${ }^{29} \sim 10^{5} \mathrm{~cm}^{2} \mathrm{~V}^{-1} \mathrm{~s}^{-1}$, the system undergoes a dimensional crossover. When the interlayer tunneling is incoherent, the electronic structure is $2 \mathrm{D}$, and we may apply the mean field calculation described above to the system. When the interlayer tunneling becomes coherent ${ }^{56}$, the electronic structure is 3D. Due to the high-mobility value of $\alpha$-(BEDT-TTF $)_{2} \mathrm{I}_{3}$, the crossover temperature is in the order of the inter-layer tunneling amplitude. We describe the inter-layer tunneling between adjacent layers with the matrix $t_{1} \mathcal{X}_{000}+t_{2} \mathcal{X}_{010}$, where the first term is the tunneling between the same molecules. The second term is the tunneling between $\mathrm{A}$ and $\mathrm{A}^{\prime}$ and between $\mathrm{B}$ and $\mathrm{C}$, where these pairs of molecules are aligned in the stacking direction. ${ }^{57}$ We note that the interlayer tunneling parametrized by $t_{2}$ is crucial to have the linear dispersion in the $z$ direction. We note that these terms do not break either the TRS or inversion symmetry, $\mathcal{I}$. Therefore, the contact points are stable against them. Because of the mirror reflection about the $a-b$ plane, two copies of each Dirac point appear at $k_{z}= \pm \pi / 2$. Since interlayer hopping parameters $t_{1}$ and $t_{2}$ are much smaller than the intralayer parameters, we include their effects based on the 2D result. We observe that the Dirac cone is tilted along the $k_{z}$ direction by $t_{1}$ as shown in Fig. 2 . For $t_{1} / t_{2}>\eta_{1}$ (Fig. 2(c) and (d)), both Dirac cones are type-II, ${ }^{28}$ which is to be applied to $\alpha$-(BEDT-TTF $)_{2} \mathrm{I}_{3}$, while for $t_{1} / t_{2}<\eta_{2}$ (Fig. 2(a) and (b)), both Dirac cones are type-I. Here, $\eta_{1}=0.6867$ and $\eta_{2}=0.6827$. Interestingly, for $\eta_{2}<t_{1} / t_{2}<\eta_{1}$, the Dirac cones at $\boldsymbol{k}_{D}^{(1)}$ with $k_{z}= \pm \pi / 2$ are type-I and the Dirac cones at $\boldsymbol{k}_{D}^{(2)}$ with $k_{z}= \pm \pi / 2$ are type-II, where we can expect the partial chiral anomaly effect to be associated with the type-I Dirac cones.

Now we consider the chiral anomaly in this system. When a magnetic field $B_{z}$ is applied along the $z$-direction, the spectrum of the Landau levels is given by

$$
\varepsilon_{n, k_{z}}^{ \pm, \tau}=-2 t_{1} \cos k_{z} \pm \sqrt{\varepsilon_{n \tau}^{2}+4 \eta_{\tau}^{2} t_{2}^{2} \cos ^{2} k_{z}},
$$

where $\varepsilon_{n \tau}$ is the $2 \mathrm{D}$ Landau level at the Dirac point $\boldsymbol{k}_{D}^{(\tau)}$ $(\tau=1,2)$ given by ${ }^{44,45}$

$$
\varepsilon_{n \tau}=\left(1-\lambda_{\tau}^{2}\right)^{3 / 4} \frac{\hbar v_{\tau}^{2 D}}{\ell_{z}} \sqrt{2|n|} .
$$

Here, $n$ is an integer, $\lambda_{\tau}$ is the tilt parameter of the Dirac cone, $v_{\tau}^{2 \mathrm{D}}$ is the averaged Fermi velocity, and $\ell_{z}=\sqrt{\hbar /\left|e B_{z}\right|}$ is the magnetic length, where $\hbar$ is the reduced Planck constant and $e$ is the electron charge. In $\alpha$-(BEDT-TTF $)_{2} \mathrm{I}_{3}, \lambda_{\tau}$ and $v_{\tau}^{2 \mathrm{D}}$ can be estimated experimentally from the analysis of the interlayer magnetoresistance. It is found that $\left(1-\lambda_{\tau}^{2}\right)^{3 / 4} v_{\tau}^{2 \mathrm{D}} \simeq 5 \times 10^{-4} \mathrm{~m} / \mathrm{s},{ }^{58}$ where $\sqrt{1-\lambda_{\tau}^{2}} \simeq 0.05 .{ }^{59}$ 
(a)
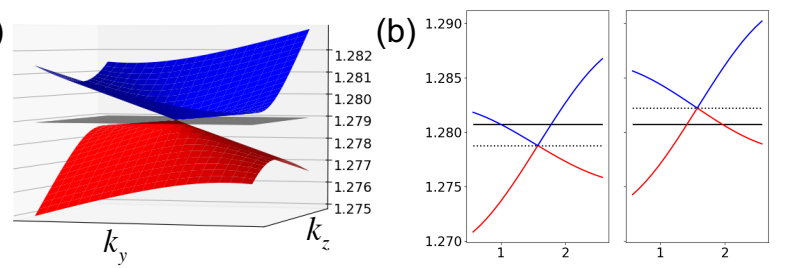

(c)

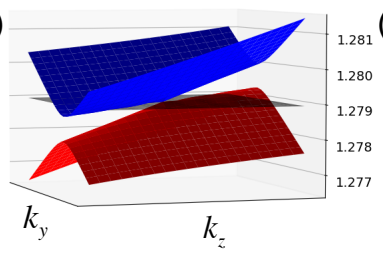

(d)

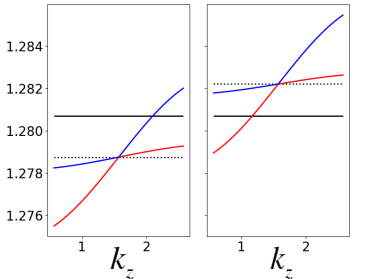

FIG. 2. (Color online) Type-I Dirac node for $t_{2} / t_{1}=4$ (a) and its energy dispersion along the $k_{z}$ axis (b). Type-II Dirac node for $t_{2} / t_{1}=1$ (c) and its energy dispersion along the $k_{z}$ axis (d). In both cases, we set $t_{1}=0.001$. The horizontal planes in (a) and (c) denote the energies of the Dirac points. In (b) and (d), the Fermi energy is denoted by the solid lines and the Dirac point energies are denoted by the dotted lines.

Since the system is a 3D Dirac semimetal, the $n=0$ Landau level has a chiral mode, $\varepsilon_{0, \pm \pi / 2+\delta k_{z}}^{ \pm, \tau}= \pm \hbar v_{z}^{\tau} \delta k_{z}$, such that

$$
v_{z}^{\tau}=\frac{2 a_{z}}{\hbar}\left(t_{1}-\eta_{\tau} t_{2}\right),
$$

where the lattice constant $a_{z}$ is explicitly shown. The plus (minus) sign is for the Dirac cone at $k_{z}=$ $\pi / 2(-\pi / 2)$. Because of the current flow between the two Dirac nodes, the negative magnetoresistance is observed when the magnetic field is tuned, within the angle $\delta \theta$, to

the direction of the inter-layer hopping, which is taken as the $z$-axis here for simplicity. We emphasize that the effect is limited to the magnetic field directions close to the inter-layer tunneling direction because of the type-II nature of the chiral anomaly. ${ }^{28}$ We note that $\delta \theta$ is approximately proportional to $1 / t_{1}$, and we find $\delta \theta \simeq 0.36^{\circ}$ when $t_{1}=0.001$.

The calculation above can be extended to include the effect of spin-orbit coupling. ${ }^{60,61}$ Due to the configuration of the molecules in the unit cell, there is no spinorbit coupling along the molecule stacking direction. We reach a clear conclusion that the spin-orbit coupling does not create any mass gap at the Dirac points. Here, the exchange correlation effect also plays a crucial role as well.

In conclusion, we have demonstrated that $\alpha$-(BEDT$\mathrm{TTF})_{2} \mathrm{I}_{3}$ is a new type of Dirac semimetal with remarkable features. Contrary to other Dirac semimetals, both the TRS and inversion are broken in a non-trivial way by bond order created by the strong inter-site electronic correlation. This result clearly extends our current understanding of the symmetry condition for Dirac semimetals. In particular, our new Dirac semimetal can be used to deepen our understanding of chiral anomaly; we expect chiral anomaly to exist in 3D but not in 2D and the transition between them can be investigated in $\alpha$-(BEDT$\mathrm{TTF})_{2} \mathrm{I}_{3}$ through the negative magnetoresistance in the direction of the inter-layer tunneling. One limitation is that the sample must be in a pressure cell, though the pressure is useful to control the electronic correlation of the system and investigate the interplay between chiral anomaly and other electronic states.

Acknowledgments. The author thanks N. Tajima for participating in helpful discussions and sharing experimental data. This work was supported by JSPS KAKENHI Grant Number JP18K18739.
* morinari.takao.5s@kyoto-u.ac.jp

1 A. Bansil, H. Lin, and T. Das, Rev. Mod. Phys. 88, 021004 (2016).

2 N. P. Armitage, E. J. Mele, and A. Vishwanath, Rev. Mod. Phys. 90, 015001 (2018).

3 A. H. Castro Neto, F. Guinea, N. M. R. Peres, K. S. Novoselov, and A. K. Geim, Rev. Mod. Phys. 81, 109 (2009).

${ }^{4}$ K. S. Novoselov, A. K. Geim, S. V. Morozov, D. Jiang, M. I. Katsnelson, I. V. Grigorieva, S. V. Dubonos, and A. A. Firsov, Nature 438, 197 (2005).

5 M. Z. Hasan and C. L. Kane, Rev. Mod. Phys. 82, 3045 (2010).

6 X.-L. Qi and S.-C. Zhang, Rev. Mod. Phys. 83, 1057 (2011).

7 Z. Wang, Y. Sun, X.-Q. Chen, C. Franchini, G. Xu, H. Weng, X. Dai, and Z. Fang, Phys. Rev. B 85, 195320 (2012).

8 Z. K. Liu, B. Zhou, Y. Zhang, Z. J. Wang, H. M. Weng, D. Prabhakaran, S.-K. Mo, Z. X. Shen, Z. Fang, X. Dai,
Z. Hussain, and Y. L. Chen, Science 343, 864 (2014).

9 Z. Wang, H. Weng, Q. Wu, X. Dai, and Z. Fang, Phys. Rev. B 88, 125427 (2013).

10 Z. K. Liu, J. Jiang, B. Zhou, Z. J. Wang, Y. Zhang, H. M. Weng, D. Prabhakaran, S.-K. Mo, H. Peng, P. Dudin, T. Kim, M. Hoesch, Z. Fang, X. Dai, Z. X. Shen, D. L. Feng, Z. Hussain, and Y. L. Chen, Nat. Mater. 13, 677 (2014).

11 A. A. Burkov, J. Phys.: Condens. Matter 27, 113201 (2015).

12 H. Nielsen and M. Ninomiya, Phys. Lett. B 130, 389 (1983).

13 S. L. Adler, Phys. Rev. 177, 2426 (1969).

14 J. S. Bell and R. Jackiw, Il Nuovo Cimento A 60, 47 (1969).

15 B. Lv, H. Weng, B. Fu, X. Wang, H. Miao, J. Ma, P. Richard, X. Huang, L. Zhao, G. Chen, Z. Fang, X. Dai, T. Qian, and H. Ding, Phys. Rev. X 5, 031013 (2015).

16 S.-M. Huang, S.-Y. Xu, I. Belopolski, C.-C. Lee, G. Chang, B. Wang, N. Alidoust, G. Bian, M. Neupane, C. Zhang, S. Jia, A. Bansil, H. Lin, and M. Z. Hasan, Nat. Commun. 
6, $7373(2015)$.

17 K. Deng, G. Wan, P. Deng, K. Zhang, S. Ding, E. Wang, M. Yan, H. Huang, H. Zhang, Z. Xu, J. Denlinger, A. Fedorov, H. Yang, W. Duan, H. Yao, Y. Wu, S. Fan, H. Zhang, X. Chen, and S. Zhou, Nat. Phys. 12, 1105 (2016).

18 N. Xu, H. M. Weng, B. Q. Lv, C. E. Matt, J. Park, F. Bisti, V. N. Strocov, D. Gawryluk, E. Pomjakushina, K. Conder, N. C. Plumb, M. Radovic, G. Autès, O. V. Yazyev, Z. Fang, X. Dai, T. Qian, J. Mesot, H. Ding, and M. Shi, Nat. Commun. 7, 11006 (2016).

19 X. Huang, L. Zhao, Y. Long, P. Wang, D. Chen, Z. Yang, H. Liang, M. Xue, H. Weng, Z. Fang, X. Dai, and G. Chen, Phys. Rev. X 5, 031023 (2015).

${ }^{20}$ J. Xiong, S. K. Kushwaha, T. Liang, J. W. Krizan, M. Hirschberger, W. Wang, R. J. Cava, and N. P. Ong, Science 350, 413 (2015).

21 C.-Z. Li, L.-X. Wang, H. Liu, J. Wang, Z.-M. Liao, and D.-P. Yu, Nat. Commun. 6, 10137 (2015).

22 C.-L. Zhang, S.-Y. Xu, I. Belopolski, Z. Yuan, Z. Lin, B. Tong, G. Bian, N. Alidoust, C.-C. Lee, S.-M. Huang, T.-R. Chang, G. Chang, C.-H. Hsu, H.-T. Jeng, M. Neupane, D. S. Sanchez, H. Zheng, J. Wang, H. Lin, C. Zhang, H.-Z. Lu, S.-Q. Shen, T. Neupert, M. Z. Hasan, and S. Jia, Nat. Commun. 7, 10735 (2016).

23 M. Hirschberger, S. Kushwaha, Z. Wang, Q. Gibson, S. Liang, C. A. Belvin, B. A. Bernevig, R. J. Cava, and N. P. Ong, Nat. Mater. 15, 1161 (2016).

24 S. M. Young, S. Zaheer, J. C. Y. Teo, C. L. Kane, E. J. Mele, and A. M. Rappe, Phys. Rev. Lett. 108, 140405 (2012).

25 J. L. Mañes, Phys. Rev. B 85, 155118 (2012).

${ }^{26}$ B.-J. Yang and N. Nagaosa, Nat. Commun. 5, 4898 (2014).

27 Z. Song, T. Zhang, and C. Fang, Phys. Rev. X 8, 031069 (2018).

28 A. A. Soluyanov, D. Gresch, Z. Wang, Q. Wu, M. Troyer, X. Dai, and B. A. Bernevig, Nature 527, 495 (2015).

29 K. Kajita, Y. Nishio, N. Tajima, Y. Suzumura, and A. Kobayashi, J. Phys. Soc. Jpn. 83, 072002 (2014).

${ }^{30}$ T. Zhang, Y. Jiang, Z. Song, H. Huang, Y. He, Z. Fang, H. Weng, and C. Fang, Nature 566, 475 (2019).

${ }^{31}$ K. Bender, I. Hennig, D. Schweitzer, K. Dietz, H. Endres, and H. J. Keller, Mol. Cryst. Liq. Cryst. 108, 359 (1984).

32 T. Mori, A. Kobayashi, Y. Sasaki, H. Kobayashi, G. Saito, and H. Inokuchi, Chem. Lett. 13, 957 (1984).

33 M. V. Kartsovnik, P. A. Kononovich, V. N. Laukin, A. G. Khomenko, and I. F. Schegolev, Sov. Phys. JETP 61, 866 (1985).

${ }^{34}$ H. Schwenk, F. Gross, C.-P. Heidmann, K. Andres, D. Schweitzer, and H. Keller, Mol. Cryst. Liq. Cryst. 119, 329 (1985).

${ }^{35}$ K. Kajita, T. Ojiro, H. Fujii, Y. Nishio, H. Kobayashi, A. Kobayashi, and R. Kato, J. Phys. Soc. Jpn. 61, 23
(1992)

36 T. Takahashi, Synth. Met. 133-134, 261 (2003).

37 H. Seo, J. Phys. Soc. Jpn. 69, 805 (2000).

38 H. Kino and H. Fukuyama, J. Phys. Soc. Jpn. 65, 2158 (1996).

39 S. Katayama, A. Kobayashi, and Y. Suzumura, J. Phys. Soc. Jpn. 75, 054705 (2006).

40 S. Ishibashi, T. Tamura, M. Kohyama, and K. Terakura, J. Phys. Soc. Jpn. 75, 015005 (2006).

41 H. Kino and T. Miyazaki, J. Phys. Soc. Jpn. 75, 034704 (2006).

42 T. Osada, J. Phys. Soc. Jpn. 77, 084711 (2008).

43 N. Tajima, S. Sugawara, R. Kato, Y. Nishio, and K. Kajita, Phys. Rev. Lett. 102, 176403 (2009).

44 T. Morinari, T. Himura, and T. Tohyama, J. Phys. Soc. Jpn. 78, 023704 (2009)

45 M. O. Goerbig, J.-N. Fuchs, G. Montambaux, and F. Piechon, Phys. Rev. B 78, 045415 (2008).

46 N. Tajima, T. Yamauchi, T. Yamaguchi, M. Suda, Y. Kawasugi, H. M. Yamamoto, R. Kato, Y. Nishio, and K. Kajita, Phys. Rev. B 88, 075315 (2013).

47 S. Moroto, K.-I. Hiraki, Y. Takano, Y. Kubo, T. Takahashi, H. M. Yamamoto, and T. Nakamura, J. Phys. IV France 114, 339 (2004).

48 A. Kobayashi, S. Katayama, Y. Suzumura, and H. Fukuyama, J. Phys. Soc. Jpn. 76, 034711 (2007).

${ }^{49}$ K. Sasaki and T. Morinari, J. Phys. Soc. Jpn. 83, 034712 (2014).

50 T. Morinari and Y. Suzumura, J. Phys. Soc. Jpn. 83, 094701 (2014).

51 (Supplemental material) The explicit form of $\mathcal{H}(\boldsymbol{k})$ is provided online.

52 K. Asano and C. Hotta, Phys. Rev. B 83, 245125 (2011).

53 F. Piéchon and Y. Suzumura, J. Phys. Soc. Jpn. 82, 033703 (2013).

54 P. Tang, Q. Zhou, G. Xu, and S.-C. Zhang, Nature Physics 12, 1100 (2016).

55 (Supplemental material) Analysises of the symmetry of the mean field Hamiltonian and the effect of $V_{c}$ are provided online.

${ }^{56}$ R. H. McKenzie and P. Moses, Phys. Rev. Lett. 81, 4492 (1998).

57 (Supplemental material) The explicit form of the Hamiltonian with three-dimensional $\boldsymbol{k}$ is provided online along with demonstration of the linear energy dispersion of the threedimensional Dirac semimetal phase for simplified cases.

58 S. Sugawara, M. Tamura, N. Tajima, R. Kato, M. Sato, Y. Nishio, and K. Kajita, J. Phys. Soc. Jpn. 79, 113704 (2010).

59 N. Tajima and T. Morinari, J. Phys. Soc. Jpn. 87, 045002 (2018).

60 S. M. Winter, K. Riedl, and R. Valentí, Phys. Rev. B 95, 060404(R) (2017).

61 T. Osada, J. Phys. Soc. Jpn. 87, 075002 (2018). 


\section{Supplementary Material}

\section{EXPLICIT FORM OF THE MEAN FIELD HAMILTONIAN}

The mean field Hamiltonian (Eq. (5) in the main text) is written as

$$
\begin{aligned}
\mathcal{H}_{\mathrm{mf}} & =\sum_{\boldsymbol{k}, \sigma, \sigma^{\prime}} c_{\boldsymbol{k} \sigma}^{\dagger}[\mathcal{H}(\boldsymbol{k})]_{\sigma \sigma^{\prime}} c_{\boldsymbol{k} \sigma^{\prime}} \\
& =\sum_{\boldsymbol{k}, \alpha, \beta, \sigma, \sigma^{\prime}} c_{\boldsymbol{k} \alpha \sigma}^{\dagger}[\mathcal{H}(\boldsymbol{k})]_{\alpha \sigma, \beta \sigma^{\prime}} c_{\boldsymbol{k} \beta \sigma^{\prime}}
\end{aligned}
$$

The matrix elements are given by

$$
\begin{aligned}
{[\mathcal{H}(\boldsymbol{k})]_{\alpha \sigma, \beta \sigma^{\prime}}=} & {\left[H_{\boldsymbol{k}}^{(+)}\right]_{\alpha \sigma, \beta \sigma^{\prime}} e^{i \boldsymbol{k} \cdot \boldsymbol{d}_{\alpha \beta}^{(+)}} } \\
& +\left[H_{\boldsymbol{k}}^{(-)}\right]_{\alpha \sigma, \beta \sigma^{\prime}} e^{-i \boldsymbol{k} \cdot \boldsymbol{d}_{\alpha \beta}^{(-)}} \\
& \left.+H_{\boldsymbol{k} \alpha \sigma}^{(c)} \delta_{\alpha \beta} \delta_{\sigma \sigma^{\prime}}\right]
\end{aligned}
$$

where

$$
\left[H_{\boldsymbol{k}}^{( \pm)}\right]_{\alpha \sigma, \beta \sigma^{\prime}}=t_{\alpha \beta}^{( \pm)}-V_{\alpha \beta} \chi_{\alpha \sigma, \beta \sigma^{\prime}, \pm}^{*}
$$

with $t_{13}^{(+)}=t_{b 3}, t_{13}^{(-)}=t_{b 2}$, etc. The on-site term is given by

$$
H_{\boldsymbol{k} \alpha \sigma}^{(c)}=2 \sum_{\gamma} V_{\alpha \gamma} n_{\gamma}+U\left\langle n_{\alpha \bar{\sigma}}\right\rangle,
$$

where $\bar{\sigma}$ is flipped to $\sigma$.

In our self-consistent calculation, we diagonalize this mean field Hamiltonian at each momentum. We take $200 \times 200$ for the number of the BZ points. $\chi_{\alpha \sigma, \beta \sigma^{\prime}, \pm}$ and $n_{\alpha \sigma}$ values are computed by calculating the expectation values, $\left\langle c_{\boldsymbol{k} \alpha \sigma}^{\dagger} c_{\boldsymbol{k} \beta \sigma^{\prime}}\right\rangle$.

\section{SYMMETRIES OF THE HAMILTONIAN AT THE DIRAC POINTS}

As discussed in the main text, the exchange correlation breaks both the TRS and the inversion symmetry. However, some components of the Hamiltonians at the Dirac points retain the inversion symmetry. To clarify this point, we represent the Hamiltonian at each Dirac point in terms of $\mathcal{X}_{\mu \nu \lambda}$, where the coefficients are as shown in Table S1. In case of $U=0.4$ and $V_{c}=V_{p}=0$, the Dirac points are located at $\boldsymbol{k}=\boldsymbol{k}_{D},-\boldsymbol{k}_{D}$ and both the TRS and the inversion symmetry are unbroken. Under these symmetries, the Hamiltonian, in general, has the form

$$
\mathcal{H}(\boldsymbol{k})=\left(\begin{array}{cccc}
a & x-i y & p & q \\
x+i y & a & p^{*} & q^{*} \\
p^{*} & p & -a+b & c \\
q^{*} & q & c & -a-b
\end{array}\right),
$$

where $a, b, c, x$, and $y$ are real functions of $\boldsymbol{k}$ and $p$ and $q$ are complex functions of $\boldsymbol{k}$. The terms $y, \operatorname{Im} p$, and $\operatorname{Im} q$ are odd, while the others are even. Based on this form, we see that some of the coefficients are the same, such as $\mathcal{X}_{020}$ and $\mathcal{X}_{320}$, and some of the coefficients have opposite signs, such as $\mathcal{X}_{200}$ and $\mathcal{X}_{210}$.

When $U=0.4, V_{c}=0.17$, and $V_{p}=0.05$, both the TRS and the inversion symmetry are broken but the symmetries in the coefficients are preserved. The symmetries are broken by the functions multiplied to $\mathcal{X}_{\mu \nu 0}$ that are neither even nor odd with respect to $\boldsymbol{k}$. Despite the presence of such symmetry breaking factors, part of the inversion symmetry is still preserved. In fact, we find

$$
\operatorname{tr}\left[\mathcal{X}_{030} \mathcal{H}\left(\boldsymbol{k}_{D}^{(1,2)}\right)\right]=-\operatorname{tr}\left[\mathcal{X}_{330} \mathcal{H}\left(\boldsymbol{k}_{D}^{(1,2)}\right)\right] .
$$

This is indispensable because $\mathcal{X}_{030}-\mathcal{X}_{330}$ and $\mathcal{X}_{300}$ combined with the identity matrix lead to degenerate and other separated levels. We note that these generators are diagonal matrices and that diagonal components arise from the charge at each molecule. Therefore, they are associated with the inversion symmetry between $n_{\mathrm{A}}$ and $n_{\mathrm{A}^{\prime}}$.

TABLE S1. Decomposition of the Hamiltonian at each Dirac point into $\mathcal{X}_{\mu \nu \lambda}$. For the case of $U=0.4$ and $V_{c}=V_{p}=0$, both the TRS and inversion symmetry are unbroken. The Dirac points are at $\boldsymbol{k}=\boldsymbol{k}_{D},-\boldsymbol{k}_{D}$, with $\boldsymbol{k}_{D}=(1.5516,-0.8044)$. Meanwhile, for the case of $U=0.4$, $V_{c}=0.17$, and $V_{p}=0.05$, these symmetries are broken, and the Dirac points are at $\boldsymbol{k}=\boldsymbol{k}_{D}^{(1)}, \boldsymbol{k}_{D}^{(2)}$, with $\boldsymbol{k}_{D}^{(1)} \neq-\boldsymbol{k}_{D}^{(2)}$, $\boldsymbol{k}_{D}^{(1)}=(0.9296,-0.8009)$, and $\boldsymbol{k}_{D}^{(2)}=(-0.9018,0.7618)$. The breaking of the TRS and the inversion symmetry is clearly illustrated through the comparison of these two cases.

\begin{tabular}{lrrrr}
\hline \hline $\mathcal{X}_{\mu \nu \lambda}$ & \multicolumn{1}{c}{$\boldsymbol{k}_{D}$} & \multicolumn{1}{c}{$-\boldsymbol{k}_{D}$} & \multicolumn{1}{c}{$\boldsymbol{k}_{D}^{(1)}$} & \multicolumn{1}{c}{$\boldsymbol{k}_{D}^{(2)}$} \\
\hline $\mathcal{X}_{010}$ & 0.0000387 & 0.0000387 & 0.0097423 & -0.0092337 \\
$\mathcal{X}_{020}$ & 0.0610626 & -0.0610626 & 0.0617559 & -0.0603957 \\
$\mathcal{X}_{030}$ & -0.0102378 & -0.0102378 & 0.0227222 & 0.0227222 \\
$\mathcal{X}_{100}$ & -0.0687389 & -0.0687389 & -0.0237383 & -0.0105359 \\
$\mathcal{X}_{110}$ & -0.0687389 & -0.0687389 & -0.0237383 & -0.0105359 \\
$\mathcal{X}_{120}$ & -0.1041172 & 0.1041172 & -0.1511936 & 0.1524187 \\
$\mathcal{X}_{130}$ & -0.0650720 & -0.0650720 & -0.1106209 & -0.1070677 \\
$\mathcal{X}_{200}$ & 0.0173256 & -0.0173256 & -0.0398026 & 0.0484009 \\
$\mathcal{X}_{210}$ & -0.0173256 & 0.0173256 & 0.0398026 & -0.0484009 \\
$\mathcal{X}_{220}$ & -0.0650720 & -0.0650720 & -0.1106209 & -0.1070677 \\
$\mathcal{X}_{230}$ & -0.1041172 & 0.1041172 & -0.1511936 & 0.1524187 \\
$\mathcal{X}_{300}$ & -0.0160446 & -0.0160446 & -0.0152960 & -0.0152960 \\
$\mathcal{X}_{310}$ & 0.0290397 & 0.0290397 & 0.0265949 & 0.0379016 \\
$\mathcal{X}_{320}$ & 0.0610626 & -0.0610626 & 0.0617559 & -0.0603957 \\
$\mathcal{X}_{330}$ & 0.0102378 & 0.0102378 & -0.0227222 & -0.0227222 \\
\hline \hline
\end{tabular}




\section{THE HAMILTONIAN WITH THREE-DIMENSIONAL WAVE VECTOR}

In the presence of the inter-layer hopping, the Hamiltonian is given by

$$
\mathcal{H}\left(k_{x}, k_{y}, k_{z}\right)=\mathcal{H}\left(k_{x}, k_{y}\right)+\left(\begin{array}{cccc}
2 t_{1} \cos k_{z} & 2 t_{2} \cos k_{z} & 0 & 0 \\
2 t_{2} \cos k_{z} & 2 t_{1} \cos k_{z} & 0 & 0 \\
0 & 0 & 2 t_{1} \cos k_{z} & 2 t_{2} \cos k_{z} \\
0 & 0 & 2 t_{2} \cos k_{z} & 2 t_{1} \cos k_{z}
\end{array}\right) \otimes s_{0} .
$$

Here, we denote dependence of $k_{x}, k_{y}$, and $k_{z}$, explicitly. The first term $\mathcal{H}\left(k_{x}, k_{y}\right)$ is defined in Eq. (S2).

As discussed in the main text, we obtain the linear energy dispersion of the three-dimensional Dirac semimetal phase from the diagonalization of Eq. (S7). However, its demonstration requires numerical calculations. In order to analytically illustrate the linear energy dispersion of the three-dimensional Dirac semimetal phase, we consider a simple model:

$$
\mathcal{H}^{(1)}\left(k_{x}, k_{y}, k_{z}\right)=\left(\begin{array}{cccc}
2 t_{1} \cos k_{z} & 2 t_{2} \cos k_{z} & v\left(k_{x}-i k_{y}\right) & 0 \\
2 t_{2} \cos k_{z} & 2 t_{1} \cos k_{z} & 0 & -v\left(k_{x}-i k_{y}\right) \\
v\left(k_{x}+i k_{y}\right) & 0 & 2 t_{1} \cos k_{z} & 2 t_{2} \cos k_{z} \\
0 & -v\left(k_{x}+i k_{y}\right) & 2 t_{2} \cos k_{z} & 2 t_{1} \cos k_{z}
\end{array}\right) \otimes s_{0}
$$

Here, $v$ is a constant. Setting $k_{z}=\pi / 2+\kappa_{z}$, we obtain

$$
E_{k_{x}, k_{y}, k_{z}}^{(1)}= \pm \sqrt{v^{2}\left(k_{x}^{2}+k_{y}^{2}\right)+4 t_{2}^{2} \sin ^{2} \kappa_{z}}-2 t_{1} \sin \kappa_{z}
$$

If $\left|\kappa_{z}\right| \ll 1$, we obtain the linear energy dispersion of the three-dimensional Dirac semimetal phase with the tilt in $k_{z}$ direction,

$$
E_{k_{x}, k_{y}, k_{z}}^{(1)}= \pm \sqrt{v^{2}\left(k_{x}^{2}+k_{y}^{2}\right)+4 t_{2}^{2} \kappa_{z}^{2}}-2 t_{1} \kappa_{z} .
$$

Note that these energy dispersions with either plus sign or minus sign are doubly degenerate along with spin degeneracy.

A more realistic model is the following Hamiltonian:

$$
\mathcal{H}^{(2)}\left(k_{x}, k_{y}, k_{z}\right)=\left(\begin{array}{cccc}
-2 t_{1} k_{z} & A+i v k_{y}-2 t_{2} k_{z} & B+v k_{x} & 0 \\
A-i v k_{y}-2 t_{2} k_{z} & -2 t_{1} k_{z} & 0 & B-v k_{x} \\
B+v k_{x} & 0 & -2 t_{1} k_{z} & -A+i k_{y}-2 t_{2} k_{z} \\
0 & B-v k_{x} & -A-i k_{y}-2 t_{2} k_{z} & -2 t_{1} k_{z}
\end{array}\right) \otimes s_{0},
$$

where $A, B, v$ are constants. Here, $\cos k_{z}$ is replaced by $-k_{z}$. The energy dispersions are obtained as follows:

$$
E_{k_{x}, k_{y}, k_{z}}^{\left(s_{1}, s_{2}\right)}=s_{1} \sqrt{A^{2}+B^{2}+v^{2}\left(k_{x}^{2}+k_{y}^{2}\right)+4 t_{2}^{2} k_{z}^{2}+2 s_{2} \sqrt{A^{2}+B^{2}} \sqrt{v^{2}\left(k_{x}^{2}+\frac{B^{2}}{A^{2}+B^{2}} k_{y}^{2}\right)+4 t_{2}^{2} k_{z}^{2}}}-2 t_{1} k_{z}
$$

with $s_{1,2}= \pm 1$. One can confirm that this is the energy dispersion of the three-dimensional Dirac semimetal phase with the tilt in $k_{z}$ direction as follows. We set

$$
\eta=\frac{1}{\sqrt{A^{2}+B^{2}}} \sqrt{v^{2}\left(k_{x}^{2}+\frac{B^{2}}{A^{2}+B^{2}} k_{y}^{2}\right)+4 t_{2}^{2} k_{z}^{2}},
$$

and

$$
a \eta=\frac{A}{A^{2}+B^{2}} v k_{y} .
$$

Equation (S12) is rewritten as

$$
\frac{E_{k_{x}, k_{y}, k_{z}}^{\left(s_{1}, s_{2}\right)}+2 t_{1} k_{z}}{\sqrt{A^{2}+B^{2}}}=s_{1} f_{s_{2}}(\eta, a),
$$


where

$$
f_{s}(\eta, a)=\sqrt{1+2 s \eta+\left(1+a^{2}\right) \eta^{2}} .
$$

Now we find

$$
\left.\frac{\partial f_{ \pm}}{\partial \eta}\right|_{\eta \rightarrow 0}= \pm 1
$$

Therefore, $E_{k_{x}, k_{y}, k_{z}}^{\left(s_{1}, s_{2}\right)}+2 t_{1} k_{z}$ is linear in $\eta$ for $\eta \ll 1$. This observation confirms that Eq. (S12) describes the energy dispersion of the three-dimensional Dirac semimetal phase with the tilt in $k_{z}$ direction.

When $v^{2}\left(k_{x}^{2}+k_{y}^{2}\right)+4 t_{2}^{2} k_{z}^{2} \ll A^{2}+B^{2}$, the approximate form is obtained as follows:

$$
E_{k_{x}, k_{y}, k_{z}}^{\left(s_{1}, s_{2}\right)} \simeq s_{1} \sqrt{A^{2}+B^{2}}+s_{2} \sqrt{v^{2}\left(k_{x}^{2}+\frac{B^{2}}{A^{2}+B^{2}} k_{y}^{2}\right)+4 t_{2}^{2} k_{z}^{2}}-2 t_{1} k_{z} .
$$

There are four energy bands and the upper two bands describe the energy dispersion of the three-dimensional Dirac semimetal phase as in $\alpha$-(BEDT-TTF $)_{2} \mathrm{I}_{3}$. However, there is an additional symmetry in this model. The lower two bands is a copy of the upper two bands. The difference is just the origin of the energy. The lower two bands are obtained by shifting the energy of the upper two bans by $2 \sqrt{A^{2}+B^{2}}$. There is no such symmetry in $\alpha$-(BEDT$\mathrm{TTF})_{2} \mathrm{I}_{3}$, though one can show that the lower two bands also have Dirac cones. 